\title{
ESTRATEGIAS PARA UNA EVALUACIÓN EFICAZ, EN EDUCACIÓN A DISTANCIA
}

\begin{abstract}
RESUMEN
La educación a distancia constituye una alternativa de formación, pero para lograr efectividad requiere de una serie de condiciones que posibiliten un desarrollo $y$ aprendizaje significativo, sin olvidar que la planificación $y$ el monitoreo son fundamentales para concretar los objetivos.
\end{abstract}

\section{ABSTRACT \\ Distance learning is an alternative way of education but it requires a series of conditions to get a significant development and learning, without forgetting that planning and monitoring are importantto fulfill the goals.}

\section{PALABRAS CLA'v E}

Educación a distancia, evaluación, calidad, estudio, TICs.

\section{KEYWORDS}

Distance learning, evaluation, quality, study, ICT.
E n el mundo globalizado en el que vivimos, se han presentado muchos avances tecnológicos que nos han brindado muchas facilidades en el comercio, en lo político, en lo social y en lo cultural, entre ellos, la educación, que ahora podemos llevarla a cabo sin la presencia física de los agentes alumno -profesor en un mismo lugar sino que a larga distancia, es su principal característica,la vía que hace posible esto es la Internet, podemos ser alumnos de universidades, tan prestigiosas como Harvard, con tan solo un clic, las tareas, evaluaciones y respuestas a cuestionarios se realizan y se envían mediante este medio. Sin embargo, me quiero referir a los resultados de estas evaluaciones, ¿son confiables? Cómo sabemos que estos trabajos y respuestas a las evaluaciones son realizados por los propios alumnos, sí muchos de ellos debido a su tiempo limitado y por la dificultad que tiene la distancia de no poder hacer las aclaración de algunas dudas que surgen en cuanto a algunos ítems y por no haber un contacto directo "cara a cara", entre tutor y alumno, ¿son realizadas adecuadamente? Por ello, me atrevo a decir que los resultados de estas evaluaciones que dan los alumnos no son confiables, debido a que las estrategias en Educación a Distancia en nuestro país todavía están en desarrollo. Lamentablemente, no asegura que los conocimientos hayan trascendido para dar paso a una Metacognición, entonces la ffducación a Distancia, estará generando profesionales de calidad? ¿Son competentes o solo son personas preocupadas en llenar de papeles su currículo, por no saber desarrollar a conciencia las evaluaciones vía Internet, generando así profesionales mediocres? ffxisten estrategias para cambiar esta realidad?, sobre todo, en los países subdesarrollados como el nuestro. Presentaré algunas propuestas hechas por diversos expertos para cambiar esta realidad que solo se puede lograr con responsabilidad y conci" ncia de lo que se va a aprender. 


\section{Educación a distancia y los cambios en su forma de evaluación}

Para empezar, quiero recurrir a la definición de Educación a Distancia "Consiste en una forma de enseñanza-aprendizaje, sin la presencia del educador y del educando en un mismo espacio físico" (Egg, 1996, p. 63). Si esta definición la unimos con lo que en la actualidad se observa, sería una educación que se desarrolla utilizando una comunicación indirecta donde el alumno y el docente están comunicados mediante el canal tecnológico que proporciona en la actualidad el medio de redes virtuales. Así lo afirma Rodríguez (2010, p. 28),"Es el aprendizaje producido a través de un medio tecnológico-digital, aunado al suministro de programas educacionales y sistemas de aprendizaje a través de medios electrónicos". Pero ¿siempre ha sido así o han surgido cambios a lo largo de los años? Martel (2004, p.18), nos comenta sobre este aspecto, que ha ido evolucionando gracias a la tecnología y no solo en su definición sino también en el aspecto evaluativo, para poder llegar al auge que hoy en día tiene, habla sobre tres generaciones: "la primera tecnología de la comunicación a distancia mediante el sistema postal y los timbres. Especialmente en Inglaterra y los Estados Unidos, era conocido como la' enseñanza por correspondencia' . $\mathrm{Su}$ metodología era una réplica de la enseñanza magisterial teniendo como modelo cognoscitivo el Instructivismo". Con respecto al último término, se refiere a una educación donde el alumno es un agente pasivo y que las evaluaciones estaban relacionadas con la información que se daba mediante preguntas cortas de manera irregulares, y solo se basaban en dar conocimientos y no verificaban si el alumno había aprendido; es decir, la verificación de los resultados en cuanto a la evaluación sumatíva era escasa, Más adelante trataré sobre la importancia de este tipo de evaluación.

la segunda generación (1950-1980),estuvo marcada, principalmente, por la inclusión de sistemas que combinaban diversos medios: escrito, radío, televisión, casettes ya sean de audio o de vídeo, teléfono, etc. Después de la Segunda Guerra Mundial, la enseñanza a distancia se transformó profundamente y cada vez más empezó a responder a los intereses y a las necesidades de una población demandante de educación permanente, en estos años se abrieron muchas instituciones de educación a distancia.

Todo esto surgió para responder a aquellas personas que querían estudiar pero por falta de tiempo no lo podían hacer de manera regular, pero qué pasaba con el aspecto evaluativo, estaban tan concentrados en la expansión de la educación a distancia que poco o nada se daba importancia a este aspecto, solo se dedicaban a crear nuevos establecimientos, mas ver los resultados de los aprendizajes en los alumnos no era de todo importantes, así lo afirma Martel (p. 11), diciendo "el modelo cognitivo vigente era principalmente conductista, acumulativo e instruccionista. En los manuales y en la notas de los cursos se incluyen ejercicios, particularmente de tipo preguntas /respuestas", con esto podemos inferir que las evaluaciones eran dadas de acuerdo a las respuestas escritas que enviaban los alumnos.

la tercera generación, siglo XXI, es la que incluye las tecnologías interactivas multídíreccionales que abren paso a una comunicación más directa entre el estudiante y el maestro, esto se da con un mejor desarrollo en países desarrollados, pero cuáles han sido las estrategias que los países subdesarrollados han tomado para que esta educación sea considera como exitosa en la actualidad. En los países l a t i noa m e r i ca n os, algunas universidades aprovechándose de este tema que está en boga con fines de expansión crearon diversos programas de educación a distancia, dejando de lado la calidad educativa, y por ende, a los resultados de la evaluación entendida como un proceso para recoger información de una realidad educativa, esto también lo afirma Rodríguez (p. 30). En este contexto, la evaluación constituye un aspecto central para analizar y corroborar la viabilidad del modelo educativo; sin embargo, dada la relativa novedad en los países latinoamericanos, las evaluaciones sobre su elaboración y efectividad son aún escasas, de ahí que la pregunta acerca de si la enseñanza en línea o a distancia es tan efectiva como la presencial continúa siendo un objeto de debate.

Por ello, muchas personas valoran más el estudio presencial que un estudio dado a distancia, ya que el grado de confiabilidad en cuanto a la educación de calidad, está deteriorado por la falta de estrategias que un programa de educación a distancia debería de tener, para que las personas puedan tener confianza en la educación que imparten.

\section{Estrategias para una evaluación eficaz}

¿Cuáles serían las principales estrategias que un programa de Educación a Distancia debería de tener en cuenta para que su calidad en el proceso de enseñanza -aprendizaje mejore? Según Cookson (2002, p. 4), se debe tener en cuenta cinco criterios, los cuales están enmarcados en el esfuerzo, la

\section{1ss | un1Fé}


ac tua ción,la capacidad, la eficiencia y el proceso.

El primero se refiere a las actividades que llevan a cabo las instituciones, donde los indicadores de este esfuerzo serían la cantidad de títulos, diplomas, certificados, los cursos diseñados, exámenes, así como las credenciales otorgadas. Está relacionado con el aspecto burocrático, papeles que confirmen que has logrado cumplir satisfactoriamente con todo lo previsto, pero esto no es un aspecto que se realiza de manera aislada, tiene que ir de la mano con el segundo punto que es el actuación o desempeño, el cual se refiere a los efectos de las actividades de la institución sobre los funcionarios . Para aplicar esto es necesario que se trate primero un diagnóstico y de acuerdo a ello se tome las medidas preventivas. El tercero está referído a la capacidad, se partiría de la siguiente pregunta IEl programa que realicé satisface las necesidades educativas y sociales? El cuarto trata sobre el costo de educación, a esto se le llama eficiencia, pero a mí parecer se debe de llamar el de la demanda ya que se promocionará aspectos profesionales según lo que pide el mercado; y el último, se refiere al proceso, es decir, la evaluación continua dela puesta en marcha de esta educación.

Rodríguez (p. 33), propone las siguientes pautas para evaluar la educación a distancia: plantea que la viabilidad de los objetos generales deben ser impartidos de acuerdo a modelos que puedan llevarse a cabo, teniendo en cuenta la realidad del contexto, donde la participación de un equipo transdíscíplínarío trabajará según su área, pero después compartirá ideas con respecto a la información que deberán detallar y evaluar para la mejora de la calidad educativa, esto debe ir a la par con las responsabilidades que cada uno debe asumir, autoevaluando su eficacia y efectividad, el diseño de los recursos y las actividades de aprendizaje deberán darse según las necesidades delos estudiantes, donde el tutor asumirá la guía de grupos pequeños con el fin de personalizar el trato con los estudiantes. El modelo debe tomar en cuenta el tiempo que dispone el estudiante para el aprendizaje electrónico, con esto se facilitará al alumno para tomar el tiempo adecuado y resolver sus tareas, prácticas y evaluaciones, conjuntamente con la guía de su tutor. También nos habla que se debe apoyar el aprendizaje independiente, donde los conocimientos teóricos que recibe mediante este canal sean llevados a la práctica. Pero, esto no podría llevarse a cabo sin una supervisión del tutor (interacción constante) y agenciarse de vídeos para poder evaluar las acciones que realiza según lo aprendido.
Uso activo, funcional y efectivo de las TIC (Tecnología de la información y la comunicación), claro está que en nuestro país el desconocimiento que hay sobre ello no permite el uso correcto de esta importante herramienta tecnológica, esto también lo afirma Monge (2007, p. 31), diciendo que" tanto los países industrializadoscomo en los países en proceso de industrialización, existen diversos·informes que indican que el uso adecuado de las TIC aún es insatisfactorio desde el punto de vista del proceso de enseñanza-aprendizaje".

Todo esto puede quedar plasmada en esta hoja, pero si no tenemos un compromiso $y$, sobre todo, responsabilidad para llevarlo a cabo utilizando, por qué no, la creatividad, de nada servirá, así como también la retroalimentación (feedback) que debe ser un ingrediente fundamental para comprobar el aprendizaje de los alumnos.

Sí tomamos en cuenta las dos posiciones en cuanto a estrategias y pautas que se deben considerar necesarias para llegar a evaluar un modelo educativo, se resumiría de la siguiente forma.

Evaluación de inicio, proceso y de final, donde el primero no se puede realizar sin antes no se ha tomado un diagnóstico; el segundo, debe estar enfocado de acuerdo a la realidad y necesidad de cada estudiante; y el tercero, que vendría a ser el final, está enfocado con el resultado de lo que se ha propuesto llegar, ya sea reforzando o concluyendo con diversas pautas que nos referirán con claridad sí hemos llegado o no a la meta o sí se necesita tomar en cuenta otros aspectos para que los resultados sean los esperados.

En mi opinión, para que estas estrategias funcionen en nuestro país, primero se debería concientizar a las personas antes de tomar un curso para ver sí realmente se sienten motivados para aprender y si están dispuestos a cumplir concíentemente con todos los trabajos y evaluaciones que se le imparten; también, debe de haber un monítoreo por parte de un profesor tutor cada cierto tiempo, y las evaluaciones deben caracterizarse por ser innovadoras, completas , que no solo el alumno se dedique a responder a cuestionarios sino que esta evaluación se realice de manera didáctica y dinámica, que estén inmiscuidos diversos rubros tanto cognitivos, afectivos, prácticos para que no solo se quede enla teoría.

Por ello, quiero enfatizar en la evaluación formativa y la sumatíva, dos Lpos de evaluaciones que se deberían aplicar en este tipo de educación, ya que 
según Scriven es una de las funciones que ayudará a conseguir las metas u objetivos propuestos, esto se dará de acuerdo a una evaluación en proceso que se ejecutará de manera continua.

La Educación a Distancia, más que llenar y llenar de conceptos teóricos a los estudian tes, debe estar enfocado también en las capacidades de reflexión y análisis de casos concretos donde se vea la reacción de las personas en los momentos, es decir debe de haber una observación permanente del tutor para que pueda haber un mayor compromiso que no sea por obligación, porque el estudiante ya ha sido concientizado por los aspectos antes mencionados.

Función sumativa, evaluación como producto, donde el análisis que haré de todos los productos recogidos se realizarán a través de un seguimiento a los alumnos que han rendido un examen. No podemos estar contentos con que haya desarrollado un examen si luego no lleva a la práctica todo lo que ha aprendido, de esa forma se determinará su valía.

Si estas dos funciones con respecto a la evaluación van de la mano con las demás propuestas, se tendrá una evaluación eficaz tanto de la educación a distancia como los resultados que esta imparte a las personas que ahora desde su casa pueden estudiar, pero implica mucho esfuerzo de ambos, tanto de alumnos como de tutores y personas que están dirigiendo esta modalidad, y que, lamentablemente

\section{REFERENCIAS}

Cookson, Peter S (2002). Acceso y equidad en la educación a distancia: Investigación, desarrollo y criterios de calidad. Revista electrónica de investigación educativa. Vol. 4, № 2. Disponible en: h ttp :/ /redi e.u a be. $\mathrm{mx} /$ vol 4 no 2 / cont enido -cookson. h tml Consultado el19/04/11.

Egg, Ander (1996). Diccionario depedagogía.Argentina: Magisterial.

Mart el, Angéline (2004). La evaluación social e individual en la era dela educación a distancia en la globalización. Revista electrónica de investigación educativa. Vol. 6, $\mathrm{N}^{\circ}$ l. Disponible en: ht tp :// redie. ens.ua be. $\mathrm{mx} /$ vol 6no 1/con ten ido - marte l.html, Consultado el08/04/11.

Monge, Julian (2007). Ventajas y desventajas de usar laboratorios virtuales en educación a distancia: la opinión del estudiantado en un proyecto de seis años de duración. Revista de Educación 31(1), 91-108. Disponible en: htt p://web.ebscohost.com/ehost / por desconociendo y mal uso de las tecnologías, no se está obteniendo los resultados que se esperan, ya que en su mayoría deciden estudiar de manera presencial en las universidades.

La concientización, motivación y monitoreo permanente, evaluaciones claras, creativas y completas que cuenten con entrevistas virtuales (web Cam) o como bien lo llama Perraton (2010, p. 5), estudios de audiencia "En algunos casos estos estudios también examinan el desempeño de los estudiantes en relación a las variables asociadas con los métodos de estudio"; es decir, agenciarse de todo aquello que facilite al tutor tomar diversos aspectos que le permitirán evaluar de manera integral al alumno, donde no solo esté enfocado a aspectos como lo pragmático, resolutivo de casos que le permitan reflexionar y así trabajar aspectos meta cognitivos. Solo así, este tipo de educación alcanzará la confiabilidad tanto de los alumnos que opten por este tipo de educación como de aquellos que están a puertas de un pre ingreso en este tipo de educación. No se puede negar que es una buena alternativa, pero nuestro país necesita realizar ciertos reajustes en cuanto a la calidad de enseñanza que imparte, sobre todo, su calidad como excelencia, prestigio ante la sociedad a nivel académico, observado en sus egresados y el resultado de un proceso que con esfuerzo de todos los agentes ejecutores, participantes y comunidad en general, lo harán posible. pdfviewer / pdfvi ew er ? si d =2clf90 32 - Sae0 - 4 3c9 -a 369 6568ec75032e $\mathrm{e}^{\circ}$ lo40s essionmgrl4\&: vid =l \&:hiclal8 Consultado el 01/06/11.

Perraton, Hilary (2000) . Repensar el programa de investigación. The internacional reviewof researc/1 in open and distance learning. Vol. 1, $\mathrm{N}^{0} \mathrm{l}$. Disponible en: http://www.irrocll.org/inclex.php/irrodl/ art icle/view/5/338Consultado el 07/06/11.

Rodríguez, Patricia (2010). Pautas para evalu ar el modelo educativo ele la licenciatura en bibliotecología y estudios de la información en la modalidad a distancia y su relación con el aprendizaje electrónico. Revista de innovación educativa. Disponible en: htt p:// web.ebscohost.com /ehost /deta il?s íd =304de5 ef-5387 444 a-ac 2c -effa 3347296 70/0 40sessíonmgr4 \&:vid= l\&: bid = 18\&:bd a ta=J mxh bmc 9 ZX M me 210Z Tlla G 9zdClsa X ZI Consultado el07/06/11.

Srta. Deysi Karín Murrieta Vallejos

Estudiante del IX ciclo del Programa Académico de Educación Especial, especialidad Retardo Mental, de la Universidad Femenina del Sagrado Corazón.

\section{1ss I un1Fé}

